\title{
Optimization of the Duty Factor of Semiconductor Light Sources Used in Photoacoustics
}

\begin{abstract}
T. STARECKI
Institute of Electronic Systems, Warsaw University of Technology

Nowowiejska 15/19, 00-665 Warsaw, Poland

There are two most common approaches to the light intensity modulation that can be found in the papers describing photoacoustic experiments. In the first one, photoacoustic signal is produced by short light pulses of relatively high power, obtained usually from pulse mode lasers. In the other, the light intensity is square or sine modulated with the duty factor of approximately $50 \%$. However, in the case of semiconductor light sources, like LED or laser diodes, it should be taken into account that duty factor of the modulation signal can be (at least in some range) exchanged with the optical output power. The paper presents discussion of the problem, including simplified theoretical analysis and experimental results. The analysis shows that in some cases, if the duty factor of a LED diode is optimized toward maximum amplitude of the induced photoacoustic signal, the resulting signal gain can be greater than $50 \%$. Optimal duty factor depends on characteristics of the particular light source, but at least in the case of LED diodes it will be usually less than the typically used $50 \%$.
\end{abstract}

PACS numbers: 43.38.Zp, 85.60.Jb

\section{Introduction}

The most common approaches to light intensity modulation in photoacoustics are pulse modulation, where photoacoustic signal is produced by short light pulses of relatively high power (usually implemented with pulse mode lasers), and continuous modulation, in which the light intensity is square or sine modulated. In both cases amplitude $A_{\mathrm{PAS}}$ of the photoacoustic signal is in a wide range proportional to the light power $P$ and quality factor $Q$ of the cell (if the cell is working in a resonance mode), and inversely proportional to the light modulation frequency $f$ and volume $V$ of the cell [1]:

$$
A_{\mathrm{PAS}} \propto \frac{P}{f V} Q .
$$

Hence, if the light source emits a light beam of constant intensity which is then 
externally modulated (by means of a chopper, electrooptic or acoustooptic modulator, etc.), the photoacoustic signal reaches maximum value when the light source works with maximum output power and modulation duty factor equals $50 \%$. However, it should be noticed that if the light source has capabilities of direct optical power modulation, which is the case of LEDs and semiconductor laser diodes, the situation is quite different.

\section{Power considerations of semiconductor light sources}

Direct modulation of the light source means that power dissipation in the light source as well as its output optical power vary with time (depending on the modulation signal). It should be noticed that most of manufacturers of semiconductor components usually allow for instantaneous exceeding of the maximum power rating, if only some additional conditions are fulfilled - e.g. that average value of the power dissipated in the component does not exceed maximum power rating. Then, if the light source is modulated with a square modulation signal, its duty factor can be decreased with (at least in some range) corresponding increase in the forward diode current, resulting finally in higher value of the emitted optical power.

\subsection{LED diodes}

In the case of LED diodes their output light power $P_{\text {out }}$ depends mainly on the forward current. Characteristics of the output light power versus forward current depends on a diode and can be strongly nonlinear (e.g. NS365L from Fig. 1), but at least the beginning part of it or in the case of some components most of the characteristics (e.g. NS375L from Fig. 1) can be with relatively low error approximated by a linear relationship

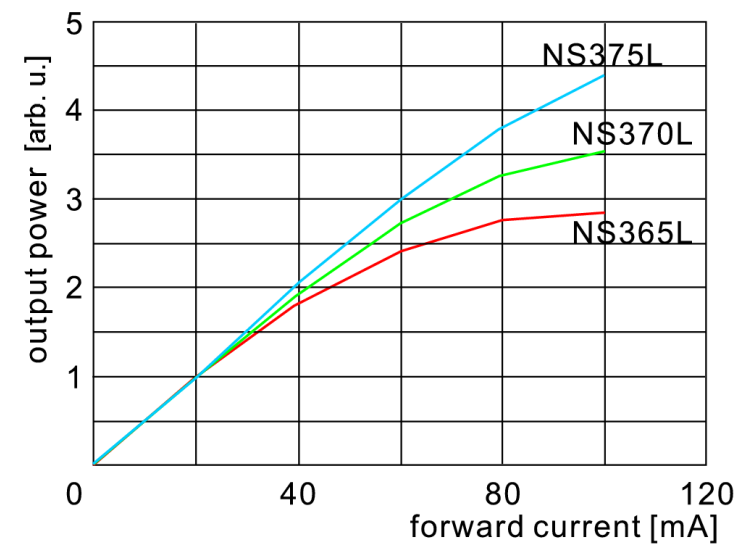

Fig. 1. Examples of optical power vs. forward current characteristics of LED diodes [2]. 


$$
P_{\text {out }} \propto I
$$

where $I$ - diode forward current. LED diodes are usually offered in plastic packages, so if the diode is modulated with a frequency of a few $\mathrm{kHz}$, its maximum instantaneous forward current is limited mainly by the maximum power dissipation, while at low modulation frequencies - by the maximum continuous forward current. To simplify evaluation it can be assumed that (even at low frequencies) instantaneous forward current can be increased with the decrease in the modulation duty factor, so that for any period $T$ :

$$
\frac{\int_{0}^{T} I(t) \mathrm{d} t}{T} \leq I_{\mathrm{CW} \max }
$$

Then, for a square wave modulation with the duty factor of $d$, maximum instantaneous forward current equals

$$
I_{\max }=\frac{I_{\mathrm{CW} \max }}{d} .
$$

For obvious reasons the current cannot be infinitely increased, and as a result characteristics $I_{\max }(d)$ given by the diode manufacturer is usually nonlinear, e.g. as in Fig. 2. However, it should be noticed that for the duty factor from the range of $25-100 \%$ the characteristics is usually not far from relationship given by Eq. (4).
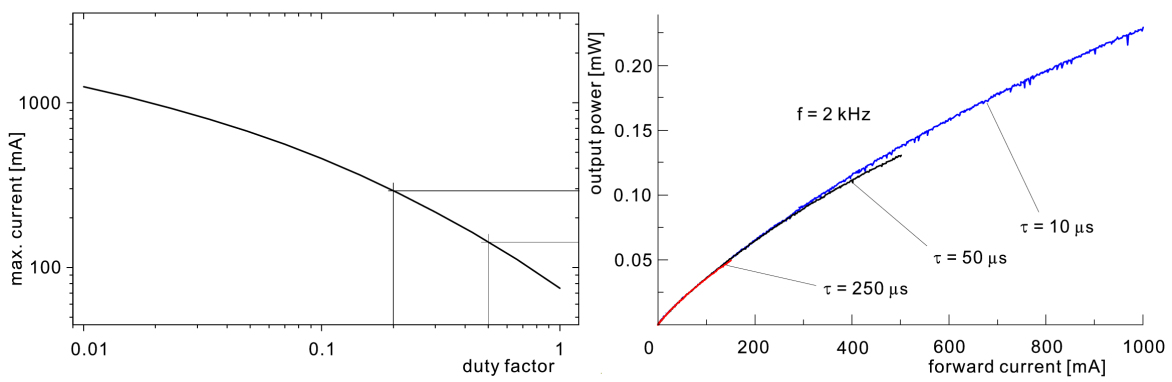

Fig. 2. Optical power vs. forward current (right) and maximum forward current vs. duty factor (left) characteristics of MIRO 42 LED diode [3].

In a square wave signal with the duty factor of $d$ and peak-to-peak value of $A$, amplitude $A_{n}$ of the $n$-th harmonic equals [4]:

$$
A_{n}=\frac{2 A}{n \pi} \sin (n \pi d) .
$$

Analyzing value of the first harmonic from Eq. (5) proves that for the light source without direct output power modulation capabilities the optimal operating point is at the duty factor of 50\%. Assuming that Eqs. (3) and (4) are valid for any value of the forward current and that output power is linearly proportional to the forward current of the diode gives 
$A_{1} \max =\frac{2}{\pi} \frac{A_{\mathrm{CW} \max }}{d} \sin (\pi d)$

which means that amplitude of the first harmonic of the induced photoacoustic signal vs. duty factor (and with the diodecurrent adjusted according to Eq. (4)) can be expressed as $(\sin x) / x$, which is maximal for the duty factor approaching zero (Fig. 3). The mentioned assumptions may lead to oversimplification, but from the given analysis and Figs. $1-3$ it is clear that:

- there is no point in increasing the duty factor over $50 \%$,

- for the duty factor within the range of 20(25)-100\% the mentioned characteristics of the LED diodes are usually close to linear relationships,

- decrease in the duty factor to 20-25\% (with corresponding increase in the instantaneous forward current) in most cases may increase the photoacoustic signal by over $40 \%$, while gain from further reduction of the duty factor is relatively low.

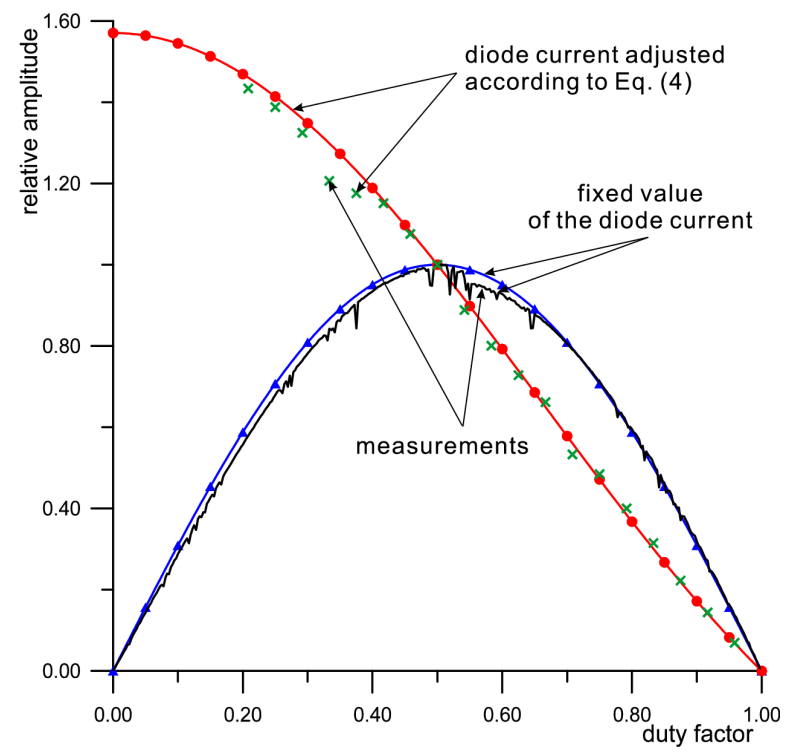

Fig. 3. Theoretical and measured characteristics of optical power vs. duty factor for fixed and adjusted (according to Eq. (4)) forward current of a LED diode (HSDL-4230).

In practice there are few semiconductor light sources that fulfill Eq. (2) and (3), and still only in a limited range of currents. An example of such a component is HSDL-4230, which has perfectly linear characteristics of optical output current vs. forward current in the range of $0-500 \mathrm{~mA}$, while the diode is rated for the maximum continuous current of $100 \mathrm{~mA}$, which means that for this diode Eq. (6) will be valid for any duty factor in the range of $20-100 \%$ at modulation frequencies 
of $2 \mathrm{kHz}$ and above (frequency limit is imposed by the manufacturer) [5]. Unfortunately, for most LED diodes $P_{\text {out }}(I)$ is nonlinear, which makes analytical solution of the optimal (in terms of duty factor) operating point of these components more difficult.

\subsection{Laser diodes}

In the case of a laser diode evaluation of its optimal operating point must be performed differently, due to some additional constraints. Laser diodes must be usually operated in a relatively small range of temperatures close or below the room temperature. Taking into consideration significant amount of power dissipated during their operation, laser diodes usually require cooling. Temperature stabilization is also needed due to temperature dependence of lasing wavelength (approximately $0.2 \mathrm{~nm} / \mathrm{K}$ ) and optical output power (Fig. 4). The latter means
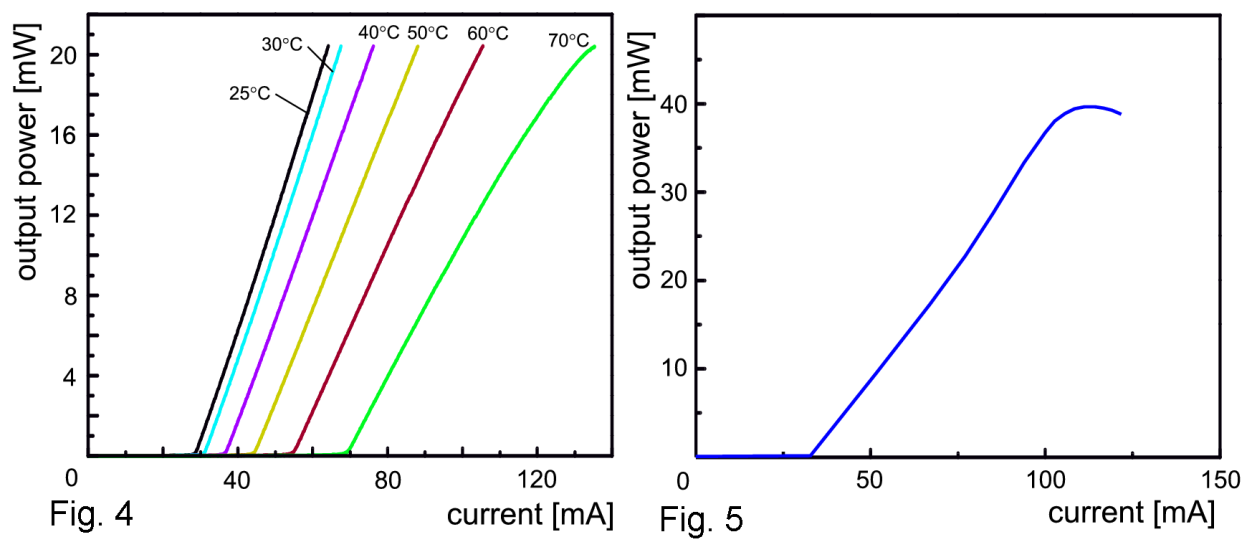

Fig. 4. Thermal dependence of optical power vs. laser diode current [6].

Fig. 5. Optical power vs. laser diode current up to the point of destruction [6].

that operating point of a laser diode cannot be expressed by a forward current value, but must be explicit given as a value of the optical output power. In the case of semiconductor laser for CW operation, value of the optical output power can be usually greater (sometimes even a few times) than rating specified in its datasheet and depends mainly on the optical power density that can be withstood by the mirrors (end facets) of the laser. However, even very short (below microsecond) and slight increase in the output optical power beyond the critical value may lead to partial or total destruction of the end facets of the laser. For this reason exceeding maximum rating of the optical power set by the manufacturer is risky and not recommended. In the case of some laser diodes the mentioned critical value of the optical power at which catastrophical optical damage occurs can be determined by cautious measuring of the laser output power vs. forward current characteristics, which is very linear within safe operation area of the laser 
and changes its slope when approaching the critical output power value (Fig. 5). Then operating point of the laser can be set at the output power slightly below the measured critical value. Taking into consideration that the mentioned optical damage is very fast, there is no option of exchanging the modulation duty factor for the output power, so that the optimal duty factor value is $50 \%$.

\section{Conclusions and remarks}

The presented analysis showed that if manufacturer of a LED diode allows for pulsed operation with optical output power higher than for $\mathrm{CW}$ mode, it is usually possible to increase photoacoustic signal by reducing the duty factor below $50 \%$. Optimal operating point (in terms of duty factor) depends directly on characteristics of the diode. In the case of HSDL-4230 it was possible to lower the duty factor to $20 \%$, which resulted in the photoacoustic signal increase by over $40 \%$.

In the case of laser diodes the main factor which determines operating point is maximum optical output power. As exchanging duty factor for the output power is usually not possible, the optimal duty factor value for semiconductor lasers is $50 \%$.

\section{References}

[1] Y.-H. Pao, Optoacoustic Spectroscopy and Detection, Academic Press, New York 1977.

[2] NS365/370/375L-CLA/CFA laser diodes datasheet, Nitride Semiconductors.

[3] MIRO-42 LED diode datasheet, Ioffe/Mirdog.

[4] W. Smith, The Scientist and Engineer's Guide to Digital Signal Processing, California Technical Publishing, San Diego 1999.

[5] HSDL-4230 LED diode datasheet, Agilent Technologies.

[6] ADL-63102 laser diode datasheet, Arima Optoelectronics. 